\title{
Futuras intenciones de emprender en estudiantes de estudios secundarios chilenos y vascos
}

\section{Jorge Torres-Ortega José Luis Monzón Campos}

RESUMEN: Las iniciativas emprendedoras -sean estas sociales o comerciales- generan un relevante impacto económico y social. Por esta razón, hoy en día apoyar al emprendimiento y a los emprendedores se ha convertido en una verdadera necesidad, ya que de ello depende en gran medida el desarrollo y crecimiento de nuestros territorios y países. En este contexto, lograr una comprensión más profunda de los principales determinantes del emprendimiento posee sin lugar a dudas un gran valor para las economías y la sociedad en general. Esto es de particular importancia en el caso de los adolescentes, por la necesidad que existe de estimular el emprendimiento desde edades tempranas. El presente artículo pretende ser un aporte en este sentido, planteándose como objetivo central caracterizar y comparar a estudiantes de estudios secundarios de dos territorios distintos desde el punto de vista de una serie de características personales y familiares, relacionándolas con sus intenciones de emprender futuras ya sea en el ámbito del emprendimiento comercial y/o social. Para el logro de tal objetivo, se aplicó una misma encuesta a una muestra de estudiantes chilenos $(\mathrm{n}=4661)$ y vascos $(\mathrm{n}=5767)$. Los resultados revelan que los estudiantes chilenos exhiben una mayor inclinación hacia seguir una carrera emprendedora que sus pares vascos. Asimismo, se observa que un conjunto de rasgos que dan forma a una "personalidad emprendedora" se asocian con mayores intenciones de emprender futuras en ambos grupos de estudiantes. Quienes desarrollan intenciones de emprender socialmente, por su parte, se vinculan más estrechamente con la posesión de sensibilidad social y sentido ético.

PALABRAS CLAVE: Intenciones emprendedoras, atributos emprendedores, emprendimiento social, emprendedor social, Chile, País Vasco. 
CLAVES ECONLIT: L26, L30, L31, L38, B55

Cómo citar este artículo/How to cite this article: TORRES-ORTEGA, J. \& MONZÓN CAMPOS, J. L. (2021): "Futuras intenciones de emprender en estudiantes de estudios secundarios chilenos y vascos", CIRIEC-España, Revista de Economía Pública, Social y Cooperativa, 103, 279314. DOI: 10.7203/CIRIEC-E.103.20933.

Correspondencia: Jorge Torres-Ortega, Universidad de Santiago de Chile, jorge.torres@ usach.cl, ORCID: 0000-0002-6640-6420; José Luis Monzón Campos, Universidad de Valencia, j.luis.monzon@uv.es, ORCID: 0000-0001-7304-4096.

ABSTRACT: Entrepreneurial initiatives -whether social or commercial- generate a significant economic and social impact. For this reason, supporting entrepreneurship and entrepreneurs has become a real necessity today, since the development and growth of our territories and countries depend to a large extent on it. In this context, achieving a deeper understanding of the main determinants of entrepreneurship is undoubtedly of great value for economies and society in general. This is particularly important in the case of adolescents, given the need to stimulate entrepreneurship from an early age. The present article aims to make a contribution in this sense, with the central objective of characterizing and comparing high school students from two different countries from the point of view of a series of personal and family characteristics, relating them to their future entrepreneurial intentions, whether in the field of commercial and/or social entrepreneurship. To achieve this objective, the same survey was applied to a sample of Chilean ( $\mathrm{n}=4661)$ and Basque $(\mathrm{n}=5767)$ students. The results reveal that Chilean students exhibit a greater inclination to follow an entrepreneurial career than their Basque peers. Likewise, it is observed that a set of traits that shape an "entrepreneurial personality" are associated with higher future entrepreneurial intentions in both groups of students. Those who develop intentions of social entrepreneurship, on the other hand, are more closely linked to the possession of social sensitivity and a sense of ethics.

KEYWORDS: Entrepreneurial intentions; entrepreneurial attributes; social entrepreneurship; social entrepreneur; Chile; Basque Country. 


\section{Expanded abstract}

\section{Future entrepreneurial intentions in Chilean and Basque high school students}

Entrepreneurial activity has been considered as one of the key factors to stimulate the development and growth of the different territories and countries around the world (GEM, 2021; Swarupa \& Goyal, 2020). This economic and social importance of entrepreneurship -both commercial and social, the latter referring to entrepreneurial activity that addresses social problems- has brought to light the need to support and promote this type of initiatives (Defourny et al., 2014; Greene, 2021). In this scenario, gaining a deeper understanding of the main determinants of entrepreneurial behaviours is undoubtedly of great value for economies and societies in general.

The literature has identified numerous factors that impact the formation of entrepreneurial intentions, which have been understood as the key predictor of the decision to start a business (Tran, 2018). One significant line a research has highlighted, for example, the relevance of individual or personal factors (Palamida, 2016). Within this tradition is the so-called demographic point of view, which assumes that it is possible to predict entrepreneurial intention in a set of individuals if they share certain traits (Palamida, 2106; Tran, 2018). At the same time, within the individual perspective several theoretical models have been developed with a focus on personality characteristics as determinants of entrepreneurial intention (Brandstatter, 2011; Zhao et al., 2010). This point of view is based on the assumption that people who decide to start a business have a different psychological profile from the rest of the population (Baron \& Henry, 2010; Rauch \& Frese, 2007).

Within the framework of this individual approach, some important questions arise: Are there differences between entrepreneurs and non-entrepreneurs in terms of their personal and family characteristics and experiences? Is it possible to distinguish between commercial and social entrepreneurs in relation to these traits? Answering these questions is of great importance because, if we know more accurately the profile of the entrepreneur -attending to this distinction between commercial and social entrepreneurs- such information could be used either to improve or create entrepreneurship training programs that take into account the -often ignored- specific training in social entrepreneurship (Bel Durán et al., 2016; GarcíaGonzález et al., 2020). This is particularly relevant in the case of the younger population, since youth is precisely the most promising source of entrepreneurial initiatives that a country can count on (Greene, 2021).

The present study seeks to make a contribution in this direction, having as its central objective to characterize and compare high school students from two different territories -Chile and the Basque Country- from the point of view of a series of personal and family characteristics, relating them to their future entrepreneurial intentions whether in the field of commercial 
and/or social entrepreneurship. Specifically, five central hypotheses are put forward: Chilean students are associated with a greater tendency to become entrepreneurs in the future (H1); work experience in enterprises, exposure to entrepreneurial role models within the family and self-perception regarding the possession of certain entrepreneurial attributes are all related to a higher probability of presenting future entrepreneurial intentions in both groups of students (H2, H3 and H4); and students with higher self-perceived levels of social sensitivity and ethical sense are linked to a stronger inclination towards social entrepreneurship (H5).

In order to achieve the main objective and test the hypotheses of this study, the same structured survey was applied to 4661 Chilean students and 5767 Basque students, all of them participants of entrepreneurship training programs in their respective educational establishments. A census sampling method was used, as it aimed to cover all the students enrolled in these entrepreneurship training modules. The survey was applied in person in both territories, and included a series of questions regarding: i) previous work experience of the students; ii) presence of entrepreneurs in their close family circle; iii) motivation for entrepreneurship and future entrepreneurial intentions; iv) characteristics of the enterprise they would create (commercial or Social Economy enterprise); and v) self-perception of entrepreneurial attributes (self-confidence, internal locus of control, risk propensity, capacity to innovate, stress tolerance, achievement motivation, social sensitivity and ethics).

Once the survey was applied and the database constructed, the analysis of the information from both territories was carried out by comparing percentages, using the chi-square coefficient to determine the association between categorical variables, and comparing means through the analysis of variance procedure.

The analysis reveals that, in accordance with what has been pointed out in the literature on entrepreneurship, there is a positive relationship between having entrepreneurs in the family and the development of future entrepreneurial intentions in both groups of students. Conversely, the positive influence of work experience on future entrepreneurial intentions is only verified in the case of Basque adolescents. The results also show that Chilean students are more inclined to follow an entrepreneurial career when compared to Basque students. These findings may be due to cultural differences related to the social valuation of entrepreneurship in both territories, where Chileans consider, in a higher proportion than Basques, that entrepreneurs enjoy a high social status (GEM, 2019a, 2019b). On the other hand, the main motivations of Chileans and Basques when it comes to entrepreneurship (difficulties in getting a job in Chile versus generating a high income in the Basque Country) are indicative of the different situations and economic expectations experienced in both places (GEM, 2019a, 2019b), which in turn can help explain the differences in entrepreneurial intentions in both populations.

With regard to entrepreneurial attributes, the results show that the perception of possessing a series of traits -which give shape to an "entrepreneurial personality"- is associated to a higher probability of presenting future entrepreneurial intentions in Chilean and Basque students. These entrepreneurial intentions, if realized, would mostly be directed towards the establishment of a mixed enterprise, that is, an organization combining social and individual objectives. In this respect, there are no major differences between Chilean and Basque stu- 
dents. The students who would incorporate social objectives in an eventual enterprise -either as priority objectives or in combination with personal objectives- are precisely those who to a greater extent declare that they possess the attributes of social entrepreneurs, in this case social sensitivity and ethics.

In sum, the results of this study demonstrate the importance of practical experiences and especially of exposure to entrepreneurial role models in shaping adolescents' future entrepreneurial intentions. They also make it possible to establish a differentiated profile between potential commercial and social entrepreneurs. All this information can be very useful in the design of entrepreneurship programs at the school level oriented to the training of commercial and/or social entrepreneurs.

A research effort such as the one proposed in this article is justified given the scarcity of studies on pre-university youth entrepreneurship, i.e., that include adolescents in school (Brüne \& Lutz, 2020; Obschonka et al., 2017). Likewise, with regard to research on social entrepreneurship, one of its central characteristics to date has been its strong emphasis on the conceptual aspect, with relatively few studies of an empirical nature and with large samples (Dacin et al., 2010; Tran, 2018). Beyond this, it should be noted that the formation of entrepreneurial intentions is a complex phenomenon, involving not only the variables considered in this research, but also others related to culture, the perception of support in the environment, etc. In this sense, this study is limited in that it covers only a small set of relevant variables. It is therefore necessary to explore in greater depth the determinants of adolescents' entrepreneurial intentions, covering, together with individual or psychological variables, factors of the broader context in which they are inserted. 


\section{Introducción}

La creación de nuevas empresas se ha considerado como uno de los factores clave para estimular el desarrollo y crecimiento de los territorios y países, ello a través de la generación de nuevos puestos de trabajo, la intensificación de la competencia en los mercados y el impulso a la innovación (Global Entrepreneurship Monitor [GEM], 2021; Swarupa \& Goyal, 2020). Por tal razón, el emprendimiento se ha convertido en un fenómeno de vital importancia para nuestras sociedades, lo que explica el gran interés que ha suscitado tanto en la teoría como en la práctica (Tiwari, 2017). Ahora bien, en el último tiempo se le ha venido brindando cada vez mayor atención a un tipo particular de emprendimiento, distinto del emprendimiento comercial o tradicional: se trata del denominado "emprendimiento social" (Dávila et al., 2021; Gupta et al., 2020; Mair \& Marti, 2006; Sánchez et al., 2018).

El creciente interés por esta variante del emprendimiento se explica en gran medida por las múltiples crisis que han aquejado a diversos territorios alrededor del mundo, lo que ha llevado a depositar grandes esperanzas en este tipo de iniciativas para la solución de los acuciantes problemas sociales que estas han traído consigo (Castro et al., 2020; Costas, 2020). Es así como, desde diferentes sectores, las empresas con fines sociales -que aquí identificamos con las entidades pertenecientes a la Economía Social- han sido consideradas como una respuesta innovadora, dinámica, inclusiva y autosostenible a los principales desafíos sociales, económicos y medioambientales que enfrentan las sociedades actuales, muchos de los cuales han sido desatendidos -ya sea por incapacidad y/o desinterés- tanto por los gobiernos y las instituciones públicas como por el sector privado (Defourny et al., 2014; Duque et al., 2021).

Es justamente en virtud del relevante impacto económico y social que generan las iniciativas emprendedoras -sean estas comerciales o sociales- que apoyar y promover el emprendimiento hoy en día se ha convertido en una necesidad (Defourny et al., 2014; Greene, 2021). Teniendo esto en cuenta, lograr una compresión más profunda de los principales determinantes de este fenómeno posee sin duda un gran valor para las economías y la sociedad en general. A pesar de la gran diversidad de niveles y enfoques desde los que se ha estudiado el emprendimiento, un aspecto destacable de la investigación en este campo ha sido la atención puesta sobre el micro-nivel de análisis (Baum et al., 2007), donde cobra importancia la comprensión del rol del individuo en el proceso emprendedor y, con ello, la identificación de los factores personales -entre ellos, y de manera clave, las características psicológicas o de personalidadde quien está detrás de una iniciativa emprendedora (Rauch \& Frese, 2007). Se trata de una perspectiva muy prolífica y de gran importancia teórica y práctica cuyo foco está puesto entonces sobre la figura capital del "emprendedor" (Hisrich et al., 2007).

Desde este punto de vista, cabe preguntarse entonces: ¿Existen diferencias entre emprendedores y no emprendedores en cuanto a sus características y experiencias personales y familiares? ¿Es posible distinguir entre emprendedores comerciales y sociales en relación con estos rasgos? Responder a estas preguntas es de gran importancia pues, si conocemos con mayor exactitud el perfil del emprendedor -atendiendo a esta distinción entre emprendedores comerciales y sociales-, dicha información podría ser utilizada ya sea para crear o mejorar 
programas ya existentes de formación de emprendedores que contemplen la -muchas veces ignorada- formación específica en emprendimiento social (Bel Durán et al., 2016; García-González et al., 2020). Esto es de especial relevancia en el caso de la población más joven, pues es precisamente la juventud la fuente más prometedora de iniciativas emprendedoras con que un país puede contar (Greene, 2021).

El presente estudio pretende ser un aporte en este sentido, planteándose como objetivo central caracterizar y comparar a estudiantes chilenos y vascos de formación profesional desde el punto de vista de una serie de características personales y familiares, relacionándolas con sus intenciones de emprender futuras ya sea en el ámbito del emprendimiento comercial y/o social. Un esfuerzo investigativo como el propuesto se justifica dada la escasez de estudios sobre emprendimiento joven preuniversitario, vale decir, que incluyan a adolescentes en etapa escolar (Brüne \& Lutz, 2020; Obschonka et al., 2017). Asimismo, en lo que respecta a la investigación sobre emprendimiento social, una de sus características centrales hasta la fecha ha sido su marcado énfasis en el aspecto conceptual, existiendo relativamente pocos estudios de carácter empírico y con muestras amplias (Dacin et al., 2010; Tran, 2018), aunque en el último tiempo han surgido importantes esfuerzos por abordar empíricamente ciertos aspectos de este fenómeno, tales como las motivaciones de los emprendedores sociales (Kim et al., 2020; Yamini et al., 2020).

El artículo se estructura de la siguiente forma: a esta introducción le sigue el apartado de marco teórico, en el que se presentan los conceptos más importantes de esta investigación, así como las hipótesis centrales que lo guían. Luego, se da cuenta de la metodología del estudio -participantes, instrumento y método de análisis-, para posteriormente exponer los principales resultados de acuerdo con los objetivos e hipótesis planteadas. Finalmente, se cierra con un apartado de discusión y conclusiones.

\section{Antecedentes}

\subsection{La intención de emprender}

El emprendimiento puede ser definido desde una perspectiva macro, o bien desde un punto de vista micro o individual; esta última perspectiva necesariamente requiere centrarse en la figura de los emprendedores, quienes son los actores clave relacionados con la identificación y explotación de oportunidades que llevan a la creación y crecimiento de empresas, vale decir, son los protagonistas del "proceso emprendedor" (Shane \& Venkataraman, 2000). En otras palabras, los comportamientos emprendedores se gatillan cuando los individuos deciden actuar sobre estas oportunidades percibidas (Shane, 2003). La investigación en emprendimiento desde una perspectiva individual se relaciona así con lo que hacen los emprendedores en términos de las actividades involucradas en el proceso de creación y crecimiento de empresas, esto es, se vincula con la pregunta respecto del por qué los individuos deciden participar en 
actividades emprendedoras y qué factores influyen en tales decisiones. De este modo, comprender al emprendedor en este sentido necesariamente exige adoptar un enfoque conductual (Carland et al., 1988), y las estructuras psicológicas que mejor exploran tales preguntas y explican los comportamientos emprendedores se relacionan con la cognición emprendedora.

La "cognición emprendedora" es la estructura de conocimiento que los individuos utilizan para tomar decisiones y hacer juicios, con el fin de identificar, evaluar y explotar una oportunidad de emprendimiento (Busenitz et al., 2003). Entre los diversos factores y procesos cognitivos que determinan el comportamiento emprendedor, la cognición emprendedora se ha centrado en las intenciones. Sobre la base de su cognición, los individuos deciden desarrollar conductas emprendedoras cuando previamente han formado fuertes intenciones de emprender; así, en esta perspectiva el emprendimiento es entendido como un proceso en donde las intenciones de los individuos son consideradas como el predictor clave de la decisión de emprender, ya sea comercial o socialmente (Mair \& Noboa, 2006). Ahora bien, en la formación de intenciones emprendedoras no solo entran en juego factores personales o psicológicos; más bien, la configuración de dichas intenciones resulta de una combinación entre estos y otros factores de contexto y situacionales (Ajzen \& Fishbein, 2005).

La literatura ha identificado numerosos factores que inciden sobre la formación de intenciones emprendedoras, particularmente dentro del ámbito del emprendimiento clásico o comercial, en desmedro del emprendimiento social (Tran, 2018). Se ha entendido que la comprensión de los procesos que conducen a la creación y crecimiento de las organizaciones empresariales requiere profundizar en las intenciones de las personas de participar en actividades de este tipo (Krueger, 2003), esto fundamentalmente por las siguientes razones: i) las intenciones de emprender representan el estado cognitivo que influye sobre el comportamiento emprendedor de los individuos (Thompson, 2009); ii) la intención de convertirse en emprendedor constituye el paso inicial que lleva luego al desarrollo de esta conducta (Kessler \& Frank, 2009); y iii) las intenciones emprendedoras proporcionan una mayor validez predictiva con respecto al comportamiento emprendedor si se las compara con las características específicas que potencialmente pueden identificarse en los emprendedores (Krueger et al., 2000).

Una de las conceptualizaciones más conocidas y utilizadas de la intención emprendedora es la que proporciona Thompson (2009: 676), quien la describe como la "convicción auto-reconocida por una persona que pretende establecer una nueva empresa comercial y conscientemente planea hacerlo en algún momento en el futuro".

A este respecto, la intención de emprender se la asocia estrechamente con la intención del individuo de crear una nueva empresa desde cero, que es justamente el foco de la presente investigación por cuanto esta considera individuos en edad temprana (estudiantes de formación profesional chilenos y vascos), de los cuales se puede esperar que no tengan mayor experiencia en el desarrollo de emprendimiento propios. Así, en este estudio se utiliza la definición de intención emprendedora de Thompson (2009), pero admitiendo la posibilidad de que los jóvenes puedan desarrollar intenciones de emprender futuras no solo respecto de empresas comerciales, sino también de empresas sociales o de la Economía Social o de empresas mixtas. 
¿Cuál es el panorama de la valoración del emprendimiento y la intención emprendedora en Chile y el País Vasco? Según datos del Global Entrepreneurship Monitor (GEM), la población adulta chilena (18-64 años), en mayor medida que la vasca, considera el emprendimiento como una opción de carrera atractiva, aunque en ambos casos estos porcentajes superan la mitad de los encuestados: 74\% y 63.2\%, respectivamente. Estas diferencias se replican en lo que respecta a la valoración social del emprendedor que existe en ambas sociedades: el $75 \%$ de los chilenos considera que los emprendedores gozan de un estatus social elevado, porcentaje que desciende al 61.3\% en la población vasca (GEM, 2019a, 2019b).

La valoración positiva del emprendimiento y los emprendedores se traduce en una alta intención de emprender en el caso chileno, no sucediendo lo mismo en el País Vasco. Así, en el año 2019 el 58\% de la población chilena adulta manifestó su intención de emprender en los próximos tres años, mientras que en Euskadi esta proporción no alcanzó a superar el 10\% (9.2\%) (GEM, 2019a, 2019b). Ahora bien, cabe señalar que los resultados referentes a intención emprendedora en el caso de la población vasca adulta contrastan con los hallazgos encontrados en el nivel de la población juvenil; de acuerdo con el IV Informe Young Business Talents (2018), el 40.8\% de los jóvenes vascos preuniversitarios prefiere emprender o montar su propia empresa como mejor opción para su futuro (37.5\% en España), frente al 38.9\% que quiere ser asalariado.

En cuanto a las motivaciones a la hora de emprender, también se observan diferencias importantes entre Chile y el País Vasco. En el caso chileno, el principal motivo para emprender se vincula a la escasez de alternativas laborales o las dificultades para conseguir un trabajo (69\%, en contraste con el 32\% de la población vasca), mientras que en Euskadi el crear riqueza o generar una renta alta es la razón aducida más común para iniciar un emprendimiento (68\%) (GEM, 2019a, 2019b).

Considerados estos antecedentes, en este estudio se plantea como hipótesis que, dadas las diferencias culturales y económicas existentes entre Chile y el País Vasco, serán los jóvenes chilenos quienes en mayor medida exhiban intenciones emprendedoras futuras (H1).

\subsection{Determinantes de las intenciones emprendedoras}

Entre las principales teorías que han abordado los determinantes psicológicos de las intenciones emprendedoras se encuentra la denominada "teoría de la personalidad"; asimismo, junto con este modelo y otros, dentro de la perspectiva individual también cabe mencionar el punto de vista demográfico (Palamida, 2016). Esta investigación se enmarca dentro de ambos enfoques.

\subsubsection{El enfoque demográfico}

Este enfoque utiliza información demográfica para dar con el "perfil tipo" del emprendedor. Se presupone que es posible predecir la intención emprendedora en un conjunto de individuos 
si estos comparten determinados rasgos. Algunas de las variables demográficas a las que se les ha prestado mayor atención en la investigación sobre emprendimiento -y que son consideradas en la presente investigación por su relevancia para la configuración de intenciones de emprender en estudiantes de estudios secundarios- son la experiencia laboral previa en empresas y los modelos de referencia emprendedores (Palamida, 2016; Tran, 2018).

La experiencia de haber trabajado en una empresa por parte de los jóvenes constituye un antecedente importante en la elección de una carrera emprendedora futura (Bretones \& Radrigán, 2018). Existen diversos estudios que señalan que la intención emprendedora y el potencial emprendedor de los individuos aumenta si estos cuentan con experiencia empresarial previa, pues esta se vincula con el aprendizaje y desarrollo de una serie de actitudes, habilidades y conocimientos que estimulan y facilitan la puesta en marcha de un emprendimiento, entre ellas, por ejemplo, la percepción de autoeficacia y deseabilidad del comportamiento emprendedor (Durán-Aponte \& Arias-Gómez, 2015; Martínez De Luco \& Campos, 2014). Es así como en el presente estudio se hipotetiza que la experiencia laboral en empresas de parte de los estudiantes chilenos y vascos se asocia con mayores intenciones de emprender futuras en estos jóvenes (H2).

En cuanto a los modelos de referencia, una serie de investigaciones han relevado la importancia del contexto y la socialización sobre la decisión que tomen los individuos respecto de la creación de una empresa en el futuro, en el sentido de que quienes han estado expuestos a un entorno con personas que hayan creado o dirigido empresas podrían llegar a desarrollar una mayor orientación hacia conductas emprendedoras que quienes no han recibido esta influencia. Esta inclinación hacia una carrera emprendedora sería posible de detectar desde la etapa adolescente (Schroder et al., 2011; Tran \& Von Korflesch, 2018). La relación entre entorno emprendedor e intenciones de emprender ha sido destacada con particular énfasis respecto del ámbito familiar. Por ejemplo, se ha establecido un estrecho vínculo entre padres emprendedores y el desarrollo de actitudes e intenciones emprendedoras en sus descendientes (Aboal \& Veneri, 2016; Begin \& Fayolle, 2014). De este modo, una de las hipótesis que se plantea en la presente investigación es que los estudiantes chilenos y vascos que hayan estado expuestos a modelos de referencia emprendedores en su círculo familiar cercano presentarán una mayor inclinación hacia emprender en el futuro (H3).

\subsubsection{La teoría de la personalidad}

Las distintas aproximaciones que vinculan rasgos de personalidad e intenciones de emprender caben dentro de lo que se ha denominado como "teoría de la personalidad" o "teoría de los rasgos", perspectiva teórica que se ha constituido como una de las líneas de investigación mejor establecidas dentro del campo de estudio de los emprendedores (Rauch \& Frese, 2007).

La teoría de la personalidad se basa en tres supuestos principales, a saber: i) El emprendimiento juega un rol central en la generación de resultados relevantes o deseables, tales como la innovación, el crecimiento económico y la creación de empleo (Reynolds et al., 2004); ii) Los emprendedores, a diferencia de otros individuos o al menos en mayor medida que el resto de 
la población, desarrollan ciertas conductas o llevan a cabo ciertas actividades o acciones que contribuyen a estos resultados deseables (Baron \& Henry, 2010); iii) Estos comportamientos y actividades están determinados -al menos parcialmente- por algunas características de personalidad (Rauch \& Frese, 2007).

Ewen (2010) señala que la personalidad se origina dentro del individuo y describe las características centrales -y relativamente estables- que dan cuenta de patrones de comportamiento consistentes. En este sentido, la personalidad incluye aspectos mentales, emocionales, sociales y físicos que son observables/no observables y conscientes/inconscientes. Los rasgos se consideran unidades fundamentales y estables, integradas en la personalidad del individuo, los cuales determinan los comportamientos individuales en una amplia variedad de situaciones. De este modo, entonces, los rasgos varían entre los individuos influyendo, consecuentemente, en sus respectivas conductas. En el ámbito específico del emprendimiento, el emprendedor ha sido determinado y diferenciado del resto de la población por un conjunto de características de personalidad y comportamientos relacionados con actividades y cursos de acción emprendedores (Rauch \& Frese, 2007). Las investigaciones se han centrado en gran medida en el papel de las características personales que configuran la personalidad de los emprendedores o la "personalidad emprendedora", y que por ende pueden predecir el comportamiento emprendedor (Rauch \& Frese, 2007).

Una línea de investigación se ha centrado en características amplias, como son los rasgos de personalidad conocidos como los Big-Five, los cuales señalan el rol de la responsabilidad, la apertura, la estabilidad emocional, la extroversión y la amabilidad en la predicción de la conducta emprendedora (Brandstatter, 2011; Wang et al., 2016). Junto con este tipo de trabajos centrados en factores generales o agregados, existe una línea de investigación más frecuente, la cual propone múltiples rasgos específicos como propios de la personalidad emprendedora. Este tipo de estudios surgen en parte como respuesta a las limitaciones que presentan los modelos basados en los Big Five, en la medida en que estos constituyen constructos excesivamente amplios, los cuales podrían predecir un conjunto general de comportamientos, pero no así conductas específicas como las que llevan a iniciar una empresa (Rauch \& Frese, 2007).

Se han llevado a cabo diversos estudios meta-analíticos que han brindado evidencia respecto de la validez predictiva de diferentes aspectos de la personalidad a la hora de analizar la conducta emprendedora (Brandstatter, 2011; Rauch \& Frese, 2007; Zhao et al., 2010). Del lado de los Big-Five, diferentes autores han señalado la existencia de un vínculo entre estos cinco rasgos de la personalidad y la conducta emprendedora, en particular de la responsabilidad y de la apertura a la experiencia (Farrington, 2012; Zhao et al., 2010). Ahora bien, Zhao et al. (2010), en su revisión meta-analítica basada en 60 estudios relacionados con los rasgos de personalidad de los Big-Five, apuntan que la magnitud de la relación es moderada. El campo de los rasgos de personalidad específicos, por otro lado, ha sido más prolífico en comparación al de los Big-Five, presentando esta perspectiva un mayor poder explicativo (Pedrosa, 2015). Las investigaciones se han centrado abrumadoramente en muestras de estudiantes universitarios (Palamida, 2016; Valencia \& Marulanda, 2019) en desmedro de otro tipo de poblaciones -por ejemplo, muestras de estudiantes escolares-, concluyendo que aquellos estudiantes que 
presentan altos niveles en rasgos tales como autoeficacia, necesidad de logro, propensión al riesgo, locus de control interno, tolerancia a la ambigüedad, necesidad de innovación, proactividad, creatividad, optimismo y tolerancia al estrés estarían más inclinados hacia el emprendimiento, esto al formar intenciones más fuertes respecto de la creación de nuevas empresas (Darmanto \& Yuliari, 2018; Newman et al., 2018; Pihie \& Bagheri, 2018). En el ámbito específico de grupos etarios de menor edad, por su parte, Frank et al. (2007) establecieron que los estudiantes escolares con mayores niveles de necesidad de logro e innovación presentan intenciones emprendedoras más fuertes.

El presente estudio se enmarca dentro del enfoque de los rasgos de personalidad específicos, en oposición a los Big-Five que representa una perspectiva más rígida. Se hipotetiza que la autopercepción respecto de la posesión de ciertos atributos emprendedores se vincula con mayores intenciones de emprender futuras en estudiantes chilenos y vascos (H4).

\subsection{El concepto de emprendimiento social y sus subconceptos}

En contraste con el emprendimiento comercial, relativamente pocas investigaciones se han abocado a determinar cuáles son los factores influyentes sobre la intención de emprender socialmente (Mair \& Noboa, 2006; Tiwari et al., 2017). Ahora bien, ¿qué es lo que se entiende específicamente por emprendimiento social? A pesar del gran interés que ha despertado el emprendimiento social en círculos académicos, profesionales y políticos, esta noción sigue siendo problemática; si bien la literatura sobre este fenómeno ha experimentado un notable desarrollo en los últimos años, aun no existe una definición unificada en torno a esta realidad económica (Dacin et al., 2010; Dávila et al., 2021; Mair \& Marti, 2006; Zahra et al., 2009).

Algo evidente cuando se habla de emprendimiento social es que no se puede hablar de él sin referirse a otros términos, al punto que muchas definiciones son inseparables, por ejemplo, de las definiciones de emprendedor social y de empresa social (Peredo \& McLean, 2006). Y es que el concepto de emprendimiento social tiene un carácter internamente complejo, el cual ha sido reconocido por varios autores, quienes lo describen como un concepto multidimensional y multifacético (Bacq \& Janssen, 2011). Así, para comprender el carácter internamente complejo del emprendimiento social, los investigadores han intentado identificar sus múltiples componentes. Se ha sugerido que la noción de emprendimiento social consta de varios componentes principales que contribuyen a su complejidad interna y que constituyen conceptos en sí mismos, entre ellos la creación de valor social, la organización del emprendimiento social y el emprendedor social (Bacq \& Janssen, 2011).

\subsubsection{Creación de valor social}

Un aspecto altamente valorado del emprendimiento social, al que se considera como un requisito previo para que un emprendimiento sea calificado como tal, es la creación de valor social (Dees, 1998; Peredo \& McLean, 2006). Este aspecto se ha abordado más a fondo en las descripciones del emprendimiento social en tanto emprendimiento que tiene principalmente 
una misión social (Dees, 1998; Sullivan-Mort et al., 2003), que crea riqueza social (Zahra et al., 2009), y que aborda problemas y necesidades sociales urgentes (Bornstein, 2004; Mair \& Marti, 2006). Por otra parte, el concepto de creación de valor social es un concepto cargado de valor e implica el "comportamiento virtuoso" (Sullivan-Mort et al., 2003), los objetivos altruistas (Tan et al., 2005) y la promoción de un propósito social que implica valores como la libertad, igualdad y tolerancia (Murphy \& Coombes, 2009).

En el presente estudio se considera que es justamente la misión social -y, por ende, la creación de valor social- el criterio más importante para distinguir el emprendimiento social del emprendimiento comercial (Mair y Marti, 2006; Peredo y McLean, 2006). En ese sentido, se entiende que los estudiantes que participan de esta investigación pueden desarrollar intenciones de emprender socialmente (primacía de los objetivos sociales), comercialmente (primacía de los fines personales, en este caso solucionar la propia estabilidad económica), o bien pueden aspirar a crear una empresa que responda a ambos tipos de objetivos.

\subsubsection{Organización del emprendimiento social (empresa social)}

Por lo general, las actividades que denominamos como emprendimiento social se organizan a lo largo del tiempo dentro de un marco organizativo. Según Mair y Marti (2006), es justamente este contexto organizativo en el que se produce el emprendimiento social el que distingue a este fenómeno de otras iniciativas dirigidas al cambio social, como son los movimientos activistas.

Tradicionalmente se ha identificado a la "empresa social" como la organización más representativa del emprendimiento social. A pesar de la falta de claridad conceptual existente respecto de lo que es la empresa social (Galera \& Borzaga, 2009; Kruse, 2020), existen ciertos puntos de consenso en la literatura sobre el tema. A menudo se entiende que la noción de empresa social -en tanto organización que provee servicios y bienes de interés general y para la cual la maximización del beneficio ya no es una condición esencial- vino a romper con el concepto clásico de empresa, es decir, representa un cuestionamiento a la concepción compartida de las empresas como organizaciones que promueven el interés exclusivo de sus propietarios (Galera \& Borzaga, 2009). En este sentido, la empresa social suele pensarse como algo "nuevo" y "distinto" respecto de los negocios clásicos y de la actividad no lucrativa tradicional, combinando en diferentes grados elementos del propósito social, la orientación de mercado y los estándares de rendimiento financiero de los negocios. Por su parte, Peattie y Morley (2008) identificaron que, a pesar de la gran cantidad de definiciones existentes en torno a este concepto, se pueden rescatar dos características definitorias de todas ellas: la centralidad del comercio (comerciar para recibir ingresos) y la primacía de los objetivos sociales.

Si bien existen muchas definiciones distintas de empresa social, es posible identificar dos tendencias o aproximaciones principales en torno a este fenómeno: la europea continental y la anglosajona o estadounidense (Galera \& Borzaga, 2009). La primera de estas corrientes -conocida como enfoque EMES- ubica a las empresas sociales dentro del ámbito específico de la Economía Social. Si bien las empresas sociales presentan ciertas características que las 
diferencian de las organizaciones típicas de la Economía Social -cooperativas, asociaciones y mutualidades-, al mismo tiempo comparten con ellas ciertos principios fundamentales -por ejemplo, su autonomía y naturaleza democrática- que vinculan a unas y otras configurando así un todo distinto tanto de las empresas del sector público como de las del sector capitalista privado (Monzón \& Chaves, 2017).

\subsubsection{Emprendedor social}

Muchos autores han considerado al emprendedor social como el elemento central del emprendimiento social (Bornstein, 2004; Dees, 1998). Del análisis de las distintas definiciones de emprendedor social se desprende que se trata de una persona que decide iniciar una empresa con una misión social explícita en mente (Dees, 1998). A diferencia del emprendedor convencional, el objetivo central de la actividad del emprendedor social es la creación de valor social en lugar de riqueza personal, abordando problemas sociales en vez de necesidades puramente individuales (Castro et al., 2020; Abu-Saifan, 2012). Asimismo, para la creación de este valor social los emprendedores sociales desarrollan actividades empresariales de maneras innovadoras, casi siempre cubriendo nichos desatendidos por gobiernos e instituciones públicas (García \& Palma, 2019; Zhara et al., 2009). La revisión de la literatura muestra que el conocimiento existente sobre el emprendedor social es mayoritariamente conceptual. Los estudios sobre emprendimiento social y emprendedores sociales se encuentran centrados principalmente en aspectos relativos a la definición y, por consiguiente, en lo conceptual más que lo empírico (Dacin et al., 2010). Con la excepción de unos pocos estudios cuantitativos, no se han llevado a cabo investigaciones con muestras amplias (Dacin et al., 2010; Kruse, 2020). En cuanto a los rasgos de personalidad que se asociarían a los emprendedores sociales -muchos de los cuales también se vinculan con los emprendedores comerciales o convencionales- se han destacado, entre otros, la autoeficacia, el compromiso, la tolerancia al riesgo, la creatividad, el carisma y el liderazgo, la proactividad, la capacidad de generar confianza y credibilidad, la amabilidad y autoexigencia en el trabajo, la fortaleza ante las dificultades, la innovación, y la sensibilidad ante la exclusión, marginación o sufrimiento de aquellos que carecen de medios económicos o influencia política (Dees, 1998; Mair \& Noboa, 2006; Sullivan-Mort et al., 2003; Zahra et al., 2009).

Si bien emprendedores sociales y comerciales comparten características comunes, un aspecto que diferencia a unos y otros se relaciona con que los primeros se vincularían en mucho mayor medida con fuertes valores sociales y altruistas tales como la sensibilidad social y un especial sentido ético, esto en virtud del tipo de fines que persiguen y por su deseo de provocar cambios significativos que sean perdurables y sustentables en el tiempo (Dacin et al., 2010; Tran, 2018). De acuerdo con esto, una de las hipótesis de esta investigación es que los estudiantes con mayores niveles autopercibidos de sensibilidad social y sentido ético serán aquellos con una inclinación más fuerte hacia el emprendimiento social (H5).

Con todos los antecedentes teóricos presentados hasta aquí, el modelo de intenciones emprendedoras desarrollado en esta investigación puede sintetizarse en la siguiente Figura: 


\section{Figura 1. Modelo de intenciones emprendedoras}

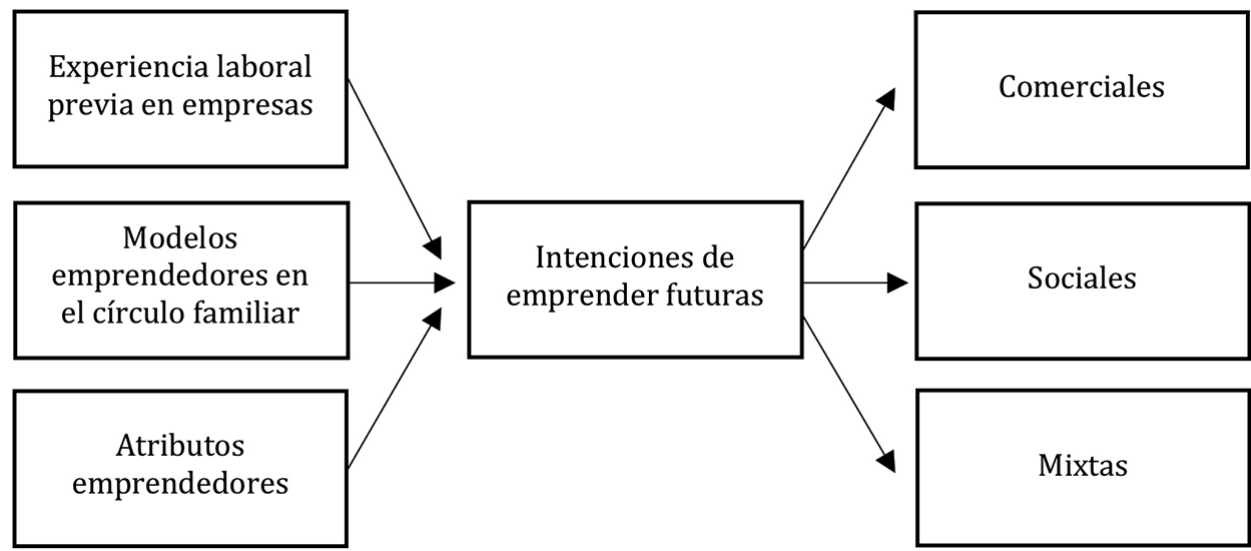

Fuente: Elaboración propia.

\section{Metodología 3.1. Participantes}

Los participantes de este estudio corresponden a estudiantes de formación profesional chilenos (de los cuatro niveles del ciclo secundario) y vascos (de grado medio y superior), todos ellos asistentes a un programa de formación en emprendimiento en sus respectivos establecimientos educacionales, por lo cual ya tienen cercanía con estos temas.

El tipo de muestreo utilizado fue de carácter censal, vale decir, se intentó cubrir a la totalidad de los estudiantes matriculados en estos módulos formativos. En Chile, los casos provienen de cuatro escuelas técnico-profesionales ubicadas en tres regiones de este país, las cuales participan de un programa de formación en emprendimiento desarrollado e implementado por la Universidad de Santiago de Chile (USACH). En el País Vasco, por su parte, los estudiantes incluidos en esta investigación pertenecen a 68 centros de formación profesional públicos y privados de todo Euskadi adscritos al programa educativo Ikasenpresa del Centro de Investigación Aplicada de la Formación Profesional del País Vasco (Tknika), que imparte el módulo "Empresa e iniciativa emprendedora".

Tanto en Chile como en el País Vasco se encuestó, durante los años 2018-2019, a más del $85 \%$ del total de estudiantes que participaron de estos programas. La muestra chilena estuvo compuesta por un total de 4661 estudiantes (72.4\% de ellos hombres y $27.6 \%$ mujeres), mientras que la muestra vasca comprendió 5767 jóvenes $(66.6 \%$ de ellos hombres y $33.4 \%$ mujeres). 


\subsection{Instrumento y método de análisis}

A los estudiantes chilenos y vascos se les aplicó una misma encuesta estructurada de elaboración propia. Dicho instrumento fue aplicado de forma presencial en Chile y en el País Vasco, e incluyó una serie de preguntas con categorías de respuesta cerradas, siendo las más relevantes para este estudio las siguientes:

- Experiencia laboral previa de los estudiantes.

- Presencia de emprendedores en su círculo familiar cercano.

- Motivación por emprender e intenciones de emprender futuras.

- Características de la empresa que crearían (comercial o de la Economía Social).

- Autopercepción de atributos emprendedores. El instrumento incluyó una escala Likert de autopercepción de atributos, la cual identifica un total de 8 características de personalidad que han sido reconocidas en la literatura como propias de los emprendedores, a saber: i) autoconfianza; ii) locus de control interno; iii) propensión al riesgo; iv) capacidad de innovar; v) tolerancia al estrés; vi) motivación de logro; vii) sensibilidad social; y viii) ética. La mayor parte de estas características han sido atribuidas al emprendedor en términos genéricos, por lo tanto, se pueden considerar como rasgos propios tanto de emprendedores comerciales como sociales. Ahora bien, y como ya se señaló, en el caso de los dos últimos atributos (sensibilidad social y ética), estos se han vinculado en mucho mayor medida con los emprendedores sociales (Dacin et al., 2010; Tran, 2018). La Tabla 1 muestra dichos atributos y las definiciones utilizadas en este estudio. 


\section{Tabla 1. Atributos emprendedores}

\begin{tabular}{ll} 
Atributo & Definición \\
\hline Autoconfianza & $\begin{array}{l}\text { Creencia personal en poseer las capacidades y habilidades necesarias para } \\
\text { iniciar una tarea y llevarla a cabo con éxito (Bandura, 1986). }\end{array}$ \\
\hline $\begin{array}{l}\text { Locus de control } \\
\text { interno }\end{array}$ & $\begin{array}{l}\text { Atribución de que la propia persona provoca y por ende controla las } \\
\text { consecuencias de sus acciones y, en cierto modo, también su destino y } \\
\text { futuro (Rotter, 1966). }\end{array}$ \\
\hline $\begin{array}{l}\text { Propensión al } \\
\text { riesgo }\end{array}$ & $\begin{array}{l}\text { Tendencia y disposición personal a asumir ciertos niveles de riesgo que } \\
\text { permitan alcanzar una meta, la cual se espera que genere mayores } \\
\text { beneficios que consecuencias negativas (Moore \& Gullone, 1996). }\end{array}$ \\
\hline $\begin{array}{l}\text { Capacidad de } \\
\text { innovar }\end{array}$ & $\begin{array}{l}\text { Voluntad e interés por buscar nuevas formas de acción, lo que implica una } \\
\text { tendencia a introducir o desarrollar nuevos productos, servicios, procesos } \\
\text { de producción, tecnologías e investigaciones (Rauch \& Frese, 2007). }\end{array}$ \\
\hline $\begin{array}{l}\text { Tolerancia al } \\
\text { estrés }\end{array}$ & $\begin{array}{l}\text { Habilidad para adaptarse y resistir los eventos adversos sin derrumbarse } \\
\text { (Brandstatter, 2011). }\end{array}$ \\
\hline Motivación de & $\begin{array}{l}\text { Impulso de sobresalir, de alcanzar metas, de esforzarse por tener éxito. } \\
\text { Aunque las personas pueden hacer las cosas por varias razones, lo que } \\
\text { está implicado en la motivación de logro es el actuar bien por sí mismo, por } \\
\text { la satisfacción intrínseca de hacerlo mejor (McClelland, 1989). }\end{array}$ \\
\hline Sensibilidad & $\begin{array}{l}\text { Sentido de obligación de asistir a personas en problemas (Volkmann et al., } \\
\text { 2012). }\end{array}$ \\
\hline Étical & $\begin{array}{l}\text { Evaluar las consecuencias y el impacto de las propias ideas, oportunidades } \\
\text { y acciones (Oliver y Galiana, 2015). }\end{array}$ \\
\hline
\end{tabular}

Fuente: Elaboración propia.

Una vez aplicada la encuesta y construida la base de datos, se desarrolló el análisis comparativo de la información de ambos territorios por medio de la comparación de porcentajes, el uso del coeficiente chi-cuadrado para determinar la asociación entre variables categóricas, y el procedimiento de análisis de varianza para comparación de medias. Todo el filtrado y análisis de datos se llevó a cabo mediante el software estadístico SPSS v.28.

\section{Resultados}

\subsection{Experiencia laboral}

Más de la mitad de los estudiantes chilenos y vascos ya cuenta con experiencia en el desarrollo de labores para la obtención de ingresos, sean estas de carácter permanente o esporádico. Ahora bien, existen diferencias importantes entre ambos grupos, con los jóvenes vascos exhibiendo mayor experiencia laboral que sus pares chilenos: $72.3 \%$ versus $51.3 \%$. 
Asimismo, en la muestra vasca se observa una mucho mayor cercanía con el mundo específicamente empresarial: el 63.9\% de los estudiantes vascos declara haber realizado trabajos o prácticas laborales en alguna empresa, lo que contrasta marcadamente con tan solo el 20.1\% observado en la muestra chilena (Figura 2).

\section{Figura 2. Experiencia laboral (\%)}

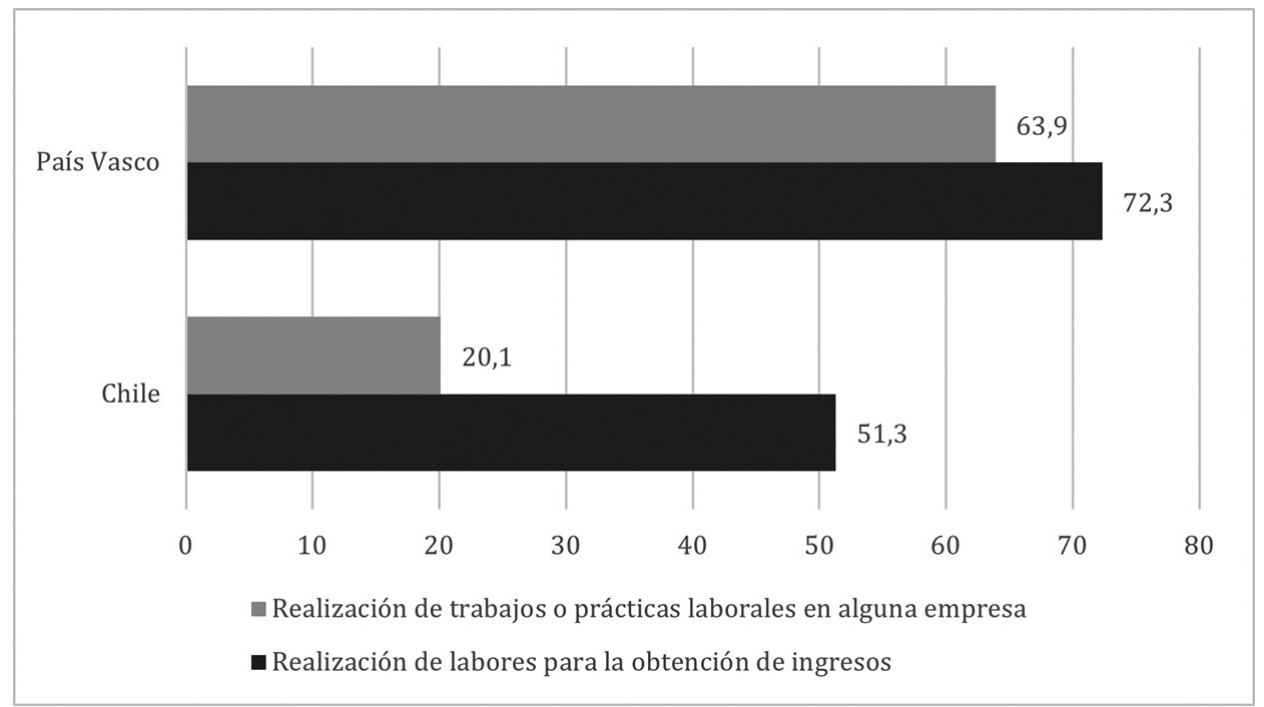

Fuente: Elaboración propia.

\subsection{Presencia de emprendedores en el círculo familiar}

Prácticamente no existen diferencias entre estudiantes chilenos y vascos en cuanto a la presencia de emprendedores en su círculo familiar cercano. En ambas muestras estos porcentajes superan ligeramente la mitad de los casos: el $52.3 \%$ de los estudiantes chilenos señala que tiene algún familiar directo (padre, madre, hermanos, tíos o abuelos) que posee un negocio propio, porcentaje que en el caso vasco alcanza el 52\% (Figura 3). 


\section{Figura 3. Emprendedores en la familia (\%)}

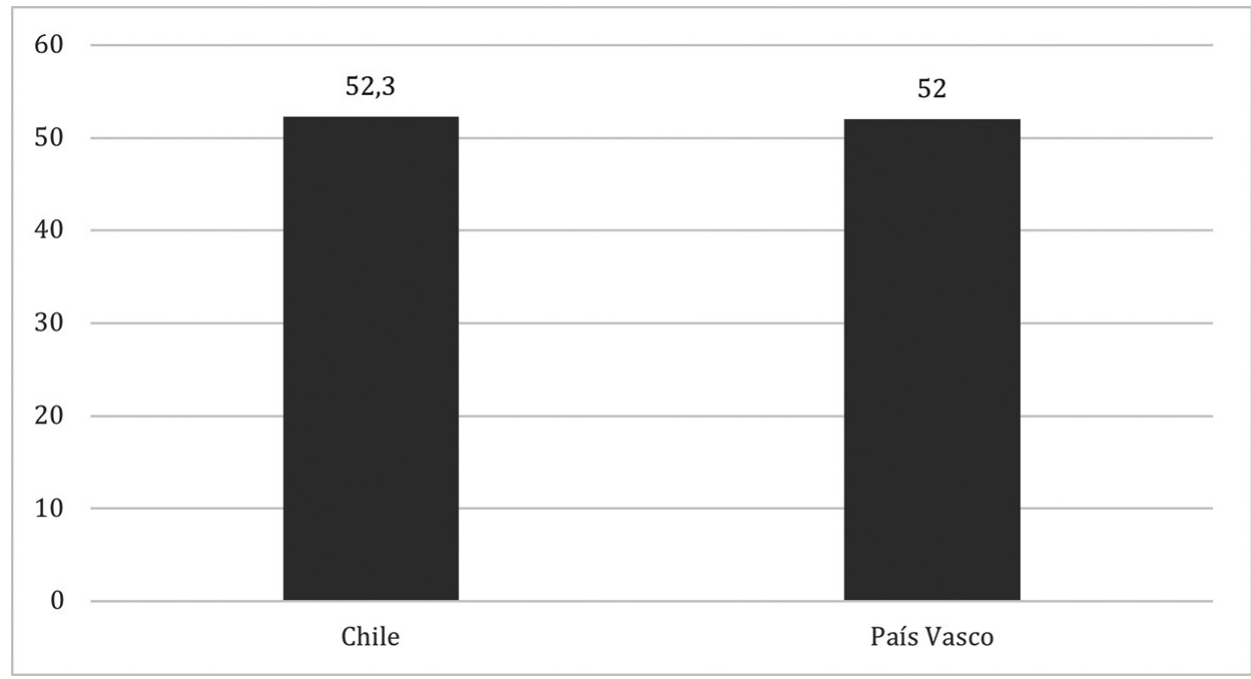

Fuente: Elaboración propia.

\subsection{Motivación por emprender e intenciones de emprender futuras}

Comparativamente, los estudiantes chilenos en mayor medida que los vascos consideran motivante tener una empresa propia (76.3\% versus 68\%) y han pensado en crear una empresa en el futuro (67.8\% versus 48.1\%) (Figura 4). Esta inclinación más fuerte de parte de los estudiantes chilenos hacia la persecución de una carrera emprendedora se ve refrendada cuando se les pide a los jóvenes que seleccionen la opción más atractiva para su futuro dentro de un conjunto de alternativas. En la muestra chilena, la opción "tener mi propia empresa" acapara la mayor cantidad de preferencias (52.5\%). Los estudiantes vascos, por su parte, preferirían mayoritariamente ser empleados de una empresa privada (35.1\%), y en segundo lugar elegirían convertirse en emprendedores (29.9\%) (Figura 5). Los datos aquí presentados apoyan entonces la H1 de esta investigación, dejando de manifiesto la mayor inclinación hacia el emprendimiento de parte de los estudiantes chilenos. 
Figura 4. Motivación por emprender e intenciones de emprender futu$\operatorname{ras}(\%)$

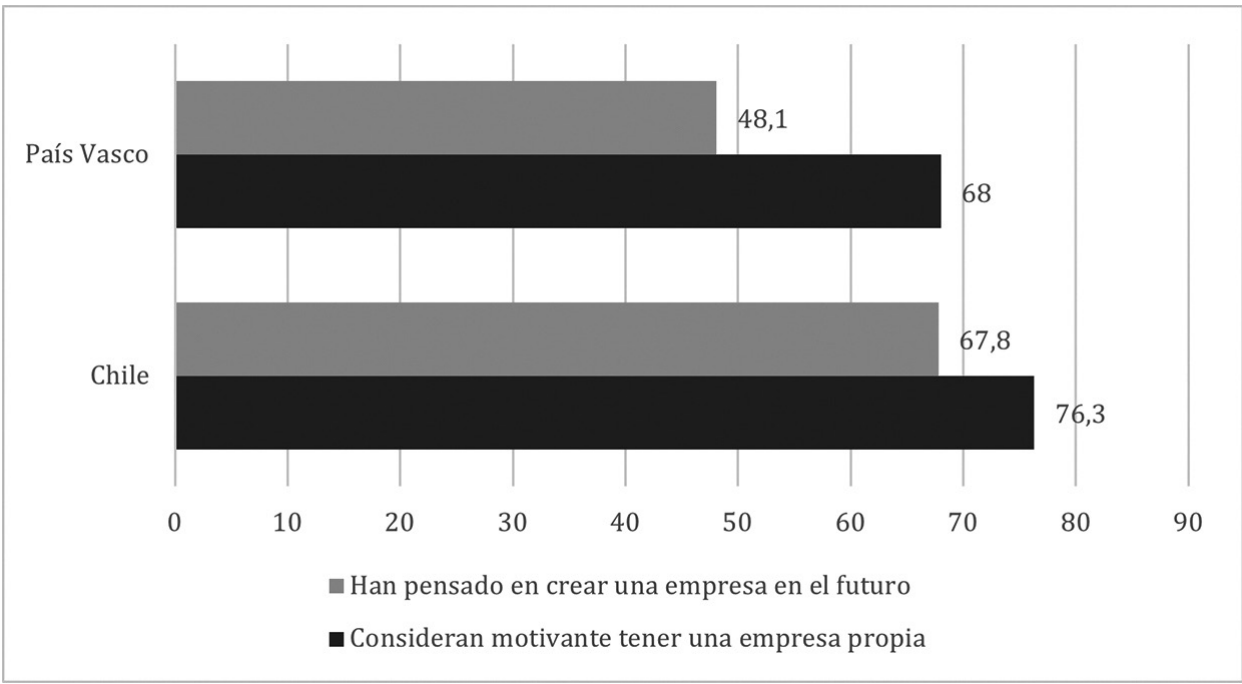

Fuente: Elaboración propia. 


\section{Figura 5. Opciones más atractivas para el futuro (\%)}

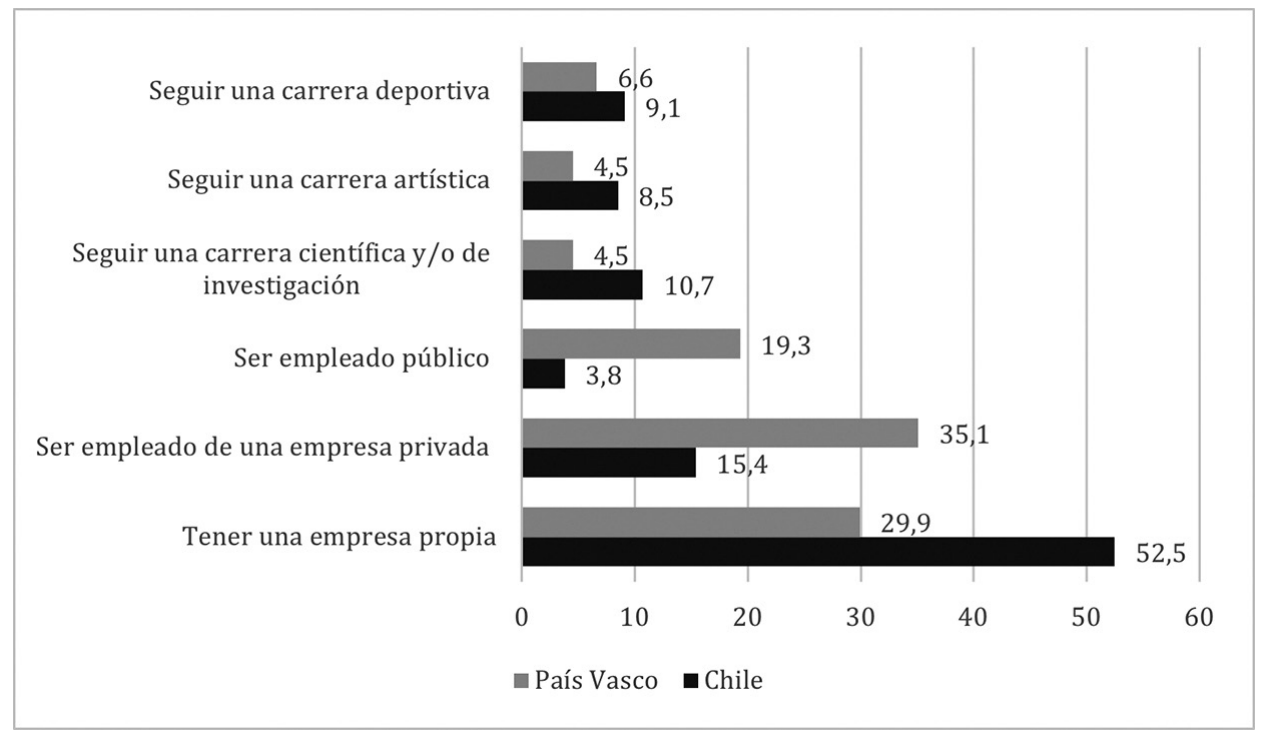

Fuente: Elaboración propia.

El análisis del estadístico chi-cuadrado $\left(\chi^{2}\right)$ revela que existe asociación entre la intención concreta de emprender en el futuro con la experiencia laboral en empresas y la presencia de emprendedores en la familia, esto tanto para Chile como para el País Vasco (sig.<0.05). En la muestra vasca, entre quienes poseen familiares emprendedores y han realizado trabajos o prácticas laborales en alguna empresa, el $67 \%$ y $57.3 \%$, respectivamente, han pensado en emprender en el futuro. La relación positiva entre tener familiares emprendedores e intención de emprender se replica en el caso chileno (57.6\%), pero no así entre esta última variable y la experiencia laboral en empresas, ya que únicamente el $22.4 \%$ de los estudiantes chilenos que han trabajado en empresas pretende emprender en el futuro (Tabla 2).

De este modo, se comprueba la H3 de relación positiva entre intenciones emprendedoras y exposición a modelos de referencia emprendedores en la familia, esto tanto en estudiantes chilenos como vascos; al contrario, la $\mathrm{H} 2$ resulta válida solo para la muestra vasca, ya que en Chile los estudiantes con pretensiones emprendedoras futuras son mayoritariamente aquellos que no cuentan con experiencia laboral en empresas. 
Tabla 2. Intención de emprender según experiencia laboral en empresas y exposición a modelos de referencia emprendedores en la familia (\%)

\begin{tabular}{lcc} 
& \multicolumn{2}{c}{$\begin{array}{c}\text { Ha pensado en llevar a cabo un } \\
\text { negocio en el futuro }\end{array}$} \\
\cline { 2 - 3 } & Chile & País Vasco \\
\hline Ha realizado trabajos o prácticas laborales en empresas & 22.4 & 67 \\
\hline Tiene familiar directo que posee negocio propio & 57.6 & 57.3 \\
\hline
\end{tabular}

Fuente: Elaboración propia.

\subsection{Características de una eventual empresa}

La mayoría de los estudiantes en Chile y en el País Vasco se inclina -en proporciones similarespor un modelo de empresa mixto, vale decir, que combine objetivos sociales e individuales. Así, el $62 \%$ y $60.4 \%$ de los jóvenes chilenos y vascos, respectivamente, declaran que si tuvieran que crear una empresa sus objetivos serían tanto solucionar un problema que beneficie a su comunidad y entorno como solucionar su propia estabilidad económica. Por otra parte, si se consideran los objetivos puramente individuales y puramente sociales, en ambas muestras existe una inclinación ligeramente superior por los primeros (Figura 6). 


\section{Figura 6. Objetivos de una eventual empresa (\%)}

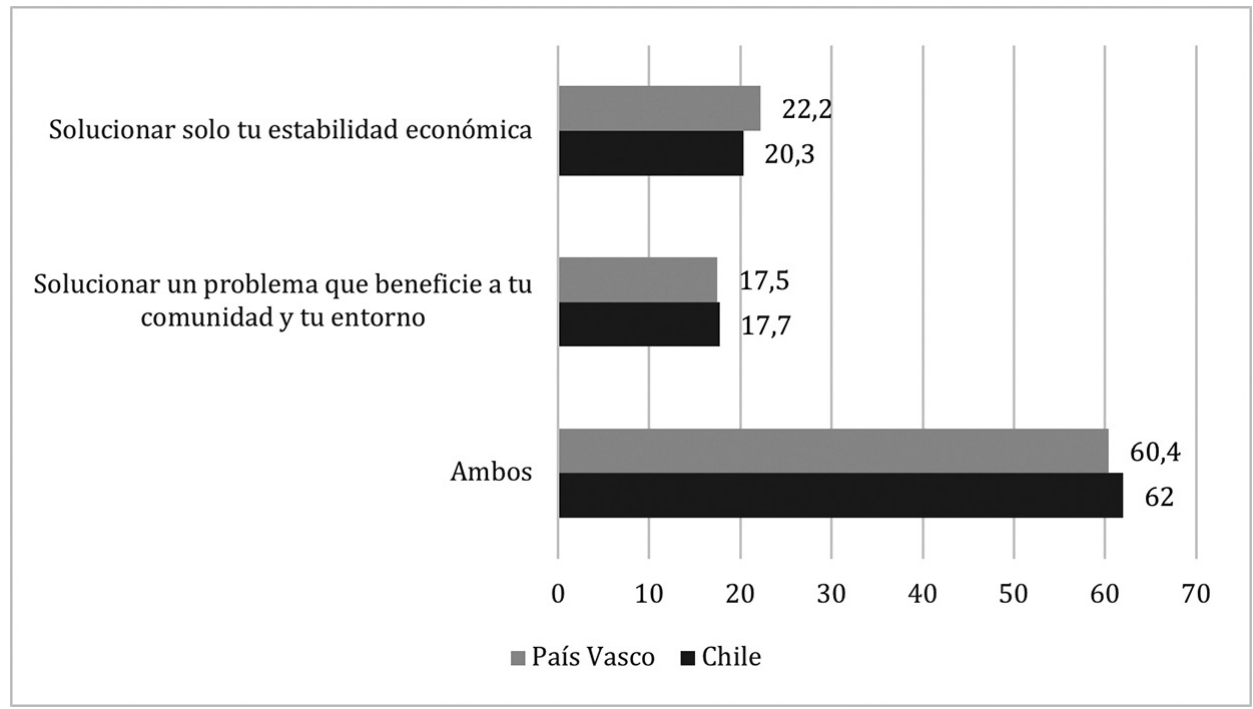

Fuente: Elaboración propia.

\subsection{Atributos emprendedores}

En la Figura 7 se registra el porcentaje de estudiantes chilenos y vascos cuya autopercepción respecto de poseer cada uno de los atributos emprendedores considerados en este estudio puede calificarse de "alta". Si bien es posible establecer que la mayor parte de los estudiantes de ambos grupos presentan una positiva valoración de sí mismos en cuanto a la posesión de estos atributos, los estudiantes chilenos destacan por sobre los vascos con porcentajes que superan el $60 \%$ en todos los rasgos, con la única excepción de la tolerancia al estrés (50.4\%). Este último atributo es también el peor valorado en el País Vasco (33\%), junto con la capacidad de innovar (48.5\%). 


\section{Figura 7. Autopercepción de atributos emprendedores (\%)}

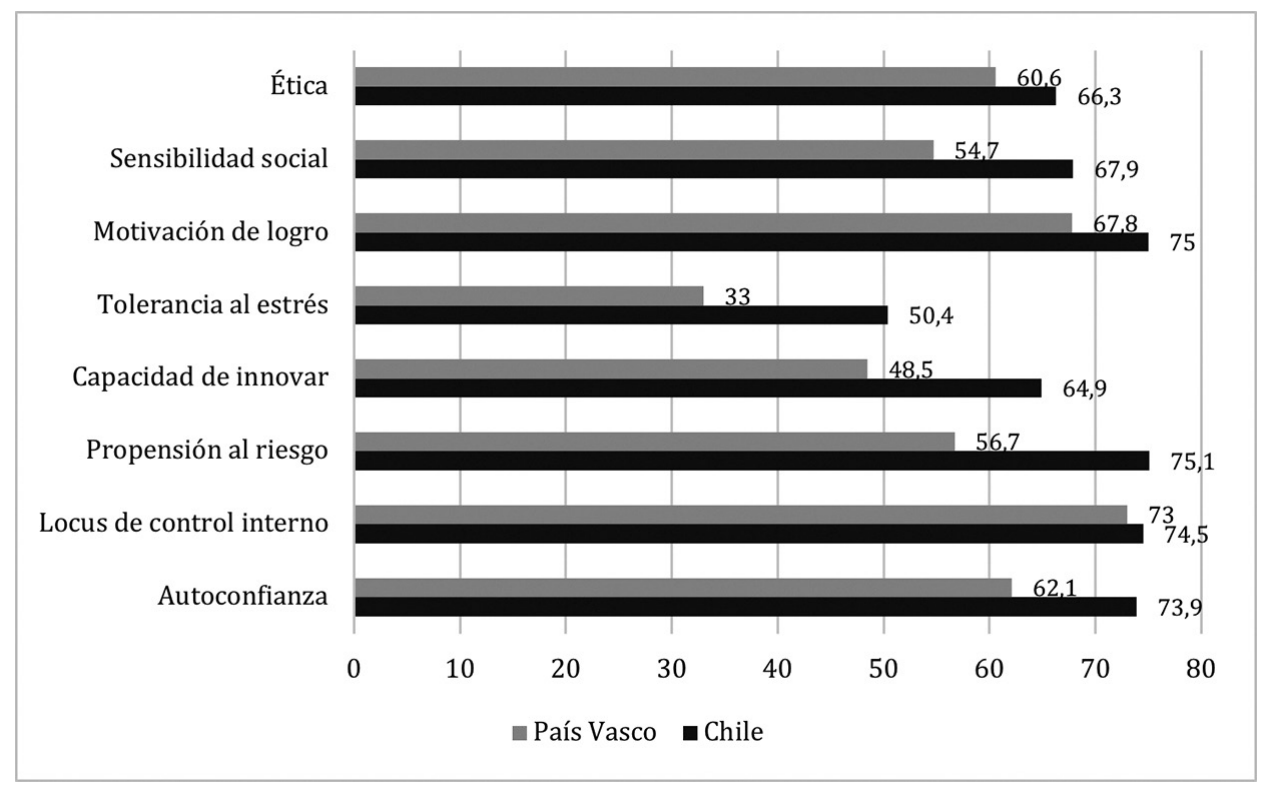

Fuente: Elaboración propia.

Por su parte, en la Tabla 3 se presentan las medias de puntaje obtenidas por los estudiantes chilenos y vascos en cada uno de los atributos emprendedores, según intenciones de emprender futuras. Se aprecia que tanto en Chile como en el País Vasco estos promedios son sistemáticamente más altos en el grupo de estudiantes que tiene pensado emprender en el futuro. El análisis de varianza arroja que las diferencias registradas en estas medias son estadísticamente significativas (sig.<0.05) (Tabla 4). De esta forma, los datos aquí presentados confirman la H4 de esta investigación, en el sentido de que los estudiantes con pretensiones de emprender futuras son al mismo tiempo los que más puntaje marcan en todos los atributos emprendedores. 
Tabla 3. Media de atributos según intención de emprender en el futuro

\begin{tabular}{|c|c|c|c|c|c|c|c|c|c|}
\hline & $\begin{array}{l}\text { Intención } \\
\text { de emp. }\end{array}$ & Autoconf. & $\begin{array}{r}\text { Locus } \\
\text { de } \\
\text { control } \\
\text { interno }\end{array}$ & $\begin{array}{l}\text { Propens. } \\
\text { al riesgo }\end{array}$ & $\begin{array}{r}\text { Capac. } \\
\text { de } \\
\text { innovar }\end{array}$ & $\begin{array}{r}\text { Toler. } \\
\text { al } \\
\text { estrés }\end{array}$ & $\begin{array}{r}\text { Motiv. } \\
\text { de } \\
\text { logro }\end{array}$ & $\begin{array}{l}\text { Sensib. } \\
\text { social }\end{array}$ & Ética \\
\hline \multirow{2}{*}{ PV } & Sí & 19.71 & 16.87 & 19.45 & 18.86 & 17.39 & 20.16 & 27.32 & 16.10 \\
\hline & No & 18.84 & 16.46 & 18.37 & 17.74 & 16.64 & 19.26 & 26.32 & 15.76 \\
\hline \multirow{2}{*}{ Chile } & Sí & 20.71 & 16.97 & 20.77 & 19.86 & 18.48 & 20.8 & 28.48 & 16.41 \\
\hline & No & 19.3 & 16.05 & 19.4 & 18.45 & 17.19 & 19.4 & 26.49 & 15.34 \\
\hline
\end{tabular}

Fuente: Elaboración propia.

Tabla 4. Análisis de varianza (atributos emprendedores e intención de emprender en el futuro)

\begin{tabular}{lcccc}
\multirow{2}{*}{ Atributos } & \multicolumn{2}{c}{ Chile } & \multicolumn{2}{c}{ País Vasco } \\
\cline { 2 - 5 } & $\mathbf{F}$ & Sig. & F & Sig. \\
\hline Autoconfianza & 165.793 & 0.000 & 111.535 & 0.000 \\
\hline Locus de control interno & 119.193 & 0.000 & 42.716 & 0.000 \\
\hline Propensión al riesgo & 161.871 & 0.000 & 164.803 & 0.000 \\
\hline Capacidad de innovar & 154.767 & 0.000 & 192.024 & 0.000 \\
\hline Tolerancia al estrés & 94.703 & 0.000 & 70.668 & 0.000 \\
\hline Motivación de logro & 155.478 & 0.000 & 117.192 & 0.000 \\
\hline Sensibilidad social & 158.865 & 0.000 & 82.712 & 0.000 \\
\hline Ética & 130.067 & 0.000 & 25.625 & 0.000 \\
\hline
\end{tabular}

Fuente: Elaboración propia.

Respecto de la autopercepción de poseer los atributos asociados en mayor medida al emprendimiento social como son la sensibilidad social y la ética, se observan diferencias importantes entre estudiantes chilenos y vascos, especialmente en relación al primero de estos rasgos. El 67.9\% de los estudiantes chilenos señala poseer este atributo, en contraste con el 54.7\% de los estudiantes vascos, lo que representa una diferencia porcentual de $13.2 \%$. En cuanto a la ética, estas diferencias son más estrechas: $66.3 \%$ de los estudiantes chilenos se considera una persona altamente ética versus el $60.6 \%$ de los jóvenes vascos, existiendo así una diferencia porcentual de 5.7\% (Figura 7). Por otra parte, el análisis de varianza revela diferencias signifi- 
cativas entre las medias de las variables dependientes "sensibilidad social" y "ética" generadas por las categorías de la variable independiente "objetivos de una eventual empresa" (Tabla 5). En el caso vasco, la prueba post-hoc de Tukey muestra que la media más alta registrada para la variable sensibilidad social se encuentra en la categoría más directamente relacionada con el emprendimiento social ("solucionar un problema social..."), la cual difiere significativamente del promedio de la categoría "solucionar tu propia estabilidad económica", pero no así de la media correspondiente a la categoría "ambas". Algo similar ocurre con la variable ética, aunque aquí el promedio más alto se observa en la categoría "ambas". Este no difiere significativamente del promedio encontrado en la categoría "solucionar un problema social...", pero sí de la media correspondiente a la categoría "solucionar tu propia estabilidad económica" (Tabla 6).

\section{Tabla 5. Análisis de varianza (sensibilidad social/ética y objetivos de una eventual empresa)}

\begin{tabular}{lcccc}
\multirow{2}{*}{ Atributos } & \multicolumn{2}{c}{ Chile } & \multicolumn{2}{c}{ País Vasco } \\
\cline { 2 - 5 } & F & Sig. & F & Sig. \\
\hline Sensibilidad social & 30.036 & 0.000 & 102.499 & 0.000 \\
\hline Ética & 25.365 & 0.000 & 60.197 & 0.000 \\
\hline
\end{tabular}

Fuente: Elaboración propia. 


\section{Tabla 6. Prueba post-hoc de Tukey (País Vasco)}

\begin{tabular}{|c|c|c|c|c|}
\hline & & & $\begin{array}{r}\text { Diferencia } \\
\text { de medias } \\
(I-J)\end{array}$ & Sig. \\
\hline \multirow{6}{*}{$\begin{array}{l}\text { Sensibilidad } \\
\text { social }\end{array}$} & \multirow{2}{*}{$\begin{array}{l}\text { Solucionar } \\
\text { un problema } \\
\text { social... }\end{array}$} & $\begin{array}{l}\text { Solucionar solo tu estabilidad } \\
\text { económica }\end{array}$ & 1.937 & 0.000 \\
\hline & & Ambas & 0.090 & 0.812 \\
\hline & \multirow{2}{*}{$\begin{array}{l}\text { Solucionar solo } \\
\text { tu estabilidad } \\
\text { económica }\end{array}$} & Solucionar un problema social... & -1.937 & 0.000 \\
\hline & & Ambas & -1.847 & 0.000 \\
\hline & \multirow[b]{2}{*}{ Ambas } & Solucionar un problema social... & -0.090 & 0.812 \\
\hline & & $\begin{array}{l}\text { Solucionar solo tu estabilidad } \\
\text { económica }\end{array}$ & 1.847 & 0.000 \\
\hline \multirow{6}{*}{ Ética } & \multirow{2}{*}{$\begin{array}{l}\text { Solucionar } \\
\text { un problema } \\
\text { social... }\end{array}$} & $\begin{array}{l}\text { Solucionar solo tu estabilidad } \\
\text { económica }\end{array}$ & 0.757 & 0.000 \\
\hline & & Ambas & -0.149 & 0.228 \\
\hline & \multirow{2}{*}{$\begin{array}{l}\text { Solucionar solo } \\
\text { tu estabilidad } \\
\text { económica }\end{array}$} & Solucionar un problema social... & -0.757 & 0.000 \\
\hline & & Ambas & -0.906 & 0.000 \\
\hline & \multirow[b]{2}{*}{ Ambas } & Solucionar un problema social... & 0.149 & 0.228 \\
\hline & & $\begin{array}{l}\text { Solucionar solo tu estabilidad } \\
\text { económica }\end{array}$ & 0.906 & 0.000 \\
\hline
\end{tabular}

Fuente: Elaboración propia.

Resultados parecidos a los vascos se pueden apreciar en la muestra chilena, con las medias más altas en ambas variables registradas en la categoría "ambas", las cuales se asemejan a los promedios de la categoría "solucionar un problema social...", pero difieren significativamente de las medias obtenidas en la categoría "solucionar tu propia estabilidad económica" (Tabla 7). En suma, respecto de las variables sensibilidad social y ética se pueden distinguir dos bloques de promedios, siendo los más altos los correspondientes a las dos categorías que incluyen objetivos sociales ("solucionar un problema social..." y "ambas") (bloque 1), los cuales se parecen entre sí y al mismo tiempo difieren significativamente de los registrados para la categoría "solucionar tu propia estabilidad económica" (bloque 2), la que contempla objetivos exclusivamente individuales. De este modo, se comprueba la $\mathrm{H} 5$ que señala que los estudiantes con mayores niveles autopercibidos de sensibilidad social y sentido ético son al mismo tiempo aquellos con una inclinación más fuerte hacia el emprendimiento social. 
Tabla 7. Prueba post-hoc de Tukey (Chile)

\begin{tabular}{|c|c|c|c|c|}
\hline & & & $\begin{array}{r}\text { Diferencia } \\
\text { de medias } \\
(I-J)\end{array}$ & Sig. \\
\hline \multirow{6}{*}{$\begin{array}{l}\text { Sensibilidad } \\
\text { social }\end{array}$} & \multirow{2}{*}{$\begin{array}{l}\text { Solucionar } \\
\text { un problema } \\
\text { social... }\end{array}$} & $\begin{array}{l}\text { Solucionar solo tu estabilidad } \\
\text { económica }\end{array}$ & 1.222 & 0.000 \\
\hline & & Ambas & -0.250 & 0.427 \\
\hline & \multirow{2}{*}{$\begin{array}{l}\text { Solucionar solo } \\
\text { tu estabilidad } \\
\text { económica }\end{array}$} & Solucionar un problema social... & -1.222 & 0.000 \\
\hline & & Ambas & -1.471 & 0.000 \\
\hline & \multirow{2}{*}{ Ambas } & Solucionar un problema social... & 0.250 & 0.427 \\
\hline & & $\begin{array}{l}\text { Solucionar solo tu estabilidad } \\
\text { económica }\end{array}$ & 1.471 & 0.000 \\
\hline \multirow{6}{*}{ Ética } & \multirow{2}{*}{$\begin{array}{l}\text { Solucionar } \\
\text { un problema } \\
\text { social... }\end{array}$} & $\begin{array}{l}\text { Solucionar solo tu estabilidad } \\
\text { económica }\end{array}$ & 0.569 & 0.000 \\
\hline & & Ambas & -0.234 & 0.121 \\
\hline & \multirow{2}{*}{$\begin{array}{l}\text { Solucionar solo } \\
\text { tu estabilidad } \\
\text { económica }\end{array}$} & Solucionar un problema social... & -0.569 & 0.000 \\
\hline & & Ambas & -0.803 & 0.000 \\
\hline & \multirow{2}{*}{ Ambas } & Solucionar un problema social... & 0.234 & 0.121 \\
\hline & & $\begin{array}{l}\text { Solucionar solo tu estabilidad } \\
\text { económica }\end{array}$ & 0.803 & 0.000 \\
\hline
\end{tabular}

Fuente: Elaboración propia.

\section{Discusión y conclusiones}

Los datos expuestos y el análisis realizado permiten delinear un perfil de los estudiantes chilenos y vascos en relación con sus intenciones de emprender futuras. En concordancia con lo que se ha señalado en la literatura sobre emprendimiento, los resultados del presente estudio muestran que existe una relación positiva entre poseer familiares emprendedores y el desarrollo de intenciones de emprender futuras. Por otra parte, se comprueba la influencia positiva de la experiencia laboral en empresas sobre las intenciones emprendedoras de los estudiantes vascos, pero no así de los chilenos; vale decir, los estudiantes chilenos desarrollan intenciones de emprender independientemente de su cercanía con empresas establecidas, lo que puede deberse en parte a la mayor prevalencia de la economía informal en este país si se lo compara con la realidad de Euskadi. 
Comparando ambas muestras, el análisis revela que son los jóvenes chilenos quienes muestran una mayor inclinación hacia seguir una carrera emprendedora que sus pares vascos. Estos hallazgos se condicen con los resultados del GEM (2019a, 2019b) para Chile y el País Vasco, los cuales, como se vio anteriormente, dejan de manifiesto importantes diferencias entre las poblaciones adultas chilena y vasca en cuanto a sus intenciones de emprender en los próximos 3 años.

Son múltiples los factores que podrían estar detrás de estas diferencias tanto a nivel adulto como adolescente. Por ejemplo, diferencias de carácter cultural relacionadas con la valoración social del emprendedor en ambos territorios. Según datos aportados por el GEM (2019a, 2019b), los chilenos consideran, en una proporción mayor que los vascos, que los emprendedores gozan de un estatus social elevado; por lo tanto, se esperaría que muchos de ellos aspirasen a convertirse en emprendedores. Por otra parte, las principales motivaciones de chilenos y vascos a la hora de emprender son indicativas de diferentes situaciones y expectativas económicas que se viven en uno y otro lugar, las cuales a su vez pueden ayudar a explicar las diferencias existentes en cuanto a intenciones emprendedoras en ambas poblaciones. En Chile, la principal razón para emprender está ligada a la escasez de alternativas laborales o las dificultades para conseguir un trabajo, a diferencia del País Vasco donde la motivación central tiene que ver con crear riqueza o generar una renta alta (GEM, 2019a, 2019b). De este modo, en el caso chileno el emprendimiento parece responder al modelo del "emprendimiento por necesidad", relacionado con lo que se han denominado motivaciones push tales como el desempleo, la idea de que "no hay mejores opciones para trabajar", etc.; al contrario, en Euskadi el emprendimiento es más bien "de oportunidad" y por ende se liga con motivaciones de tipo pull, como son el deseo de ser independiente, las posibilidades de desarrollo personal, etc. (Verheul et al., 2016).

En cuanto a los atributos emprendedores, en esta investigación se comprueba que la posesión de una serie de rasgos -que dan forma a una "personalidad emprendedora"- se asocia con mayores intenciones de emprender futuras en los estudiantes chilenos y vascos. Se observa una autovaloración altamente positiva de parte de los estudiantes, especialmente de los chilenos, en relación con estos atributos, lo que se condice con los resultados del GEM (2019a, $2019 \mathrm{~b}$ ) en cuanto a que el $76 \%$ de los chilenos y el $50.3 \%$ de los vascos considera que posee las capacidades necesarias para emprender. De este modo, las tres variables del modelo planteado en esta investigación muestran tener influencia sobre las intenciones de emprender de los estudiantes, aunque una de ellas -la experiencia laboral en empresas- solo de forma parcial.

Estas intenciones de emprender, de concretarse, se volcarían mayoritariamente hacia el establecimiento de una empresa mixta, vale decir, una organización empresarial donde se combinen objetivos sociales e individuales. En esto no hay mayores diferencias entre estudiantes chilenos y vascos. Los estudiantes que incorporarían objetivos sociales en una eventual empresa -ya sea como objetivos prioritarios, o bien en combinación con objetivos personalesson justamente aquellos que en mayor medida declaran poseer atributos propios de los emprendedores sociales, en este caso la sensibilidad social y la ética. 
La presente investigación se ha centrado en factores personales, psicológicos y familiares para explicar las intenciones de emprender de estudiantes de estudios secundarios en dos contextos distintos; ahora bien, la formación de intenciones emprendedoras es un fenómeno complejo, donde intervienen no solo esta clase de variables, sino que también otras relacionadas con la cultura -por ejemplo, la existencia o no de una "cultura emprendedora"-, la percepción de apoyos en el entorno -o la presencia de un "ecosistema de emprendimiento" adecuado-, etc. Este estudio presenta la limitación de que abarca solo un pequeño conjunto de variables relevantes. Es necesario entonces que se exploren en mayor profundidad los determinantes de las intenciones de emprender de los adolescentes, abarcando, junto con las variables individuales o psicológicas, factores del contexto más amplio en donde estos se encuentran insertos. De este esfuerzo depende la creación de programas escolares de formación en emprendimiento efectivos, los cuales también contemplen la educación en emprendimiento social.

\section{Bibliografía}

ABOAL, D. \& VENERI, F. (2016): “Entrepreneurs in Latin America”, Small Business Economics, 46, 503-525, DOI: $10.1007 / \mathrm{s} 11187-015-9696-3$

ABU-SAIFAN, S. (2012): "Social entrepreneurship: Definition and boundaries", Technology Innovation Management Review, February, 22-27, DOI: 10.22215/TIMREVIEW/523

AJZEN, I. \& FISHBEIN, M. (2005): "The influence of attitudes on behavior", en D. ALBARRACIN, B. JOHNSON \& M. ZANNA (Eds.), The handbook of attitudes (pp. 173-221), Mahwah, NJ: Lawrence Erlbaum Associates.

BACQ, S. \& JANSSEN, F. (2011): "The multiple faces of social entrepreneurship: A review of definitional issues based on geographical and thematic criteria", Entrepreneurship \& Regional Development, 23(5-6), 373-403, DOI: 10.1080/08985626.2011.577242

BANDURA, A. (1986): Social foundation of thought and action: A social cognitive theory, Englewood Cliffs, NJ: Prentice-Hall.

BARON, R. \& HENRY, R.A. (2010): "Entrepreneurship: The genesis of organizations", en S. ZEDECK (Ed.), APA handbook of industrial and organizational psychology (pp- 241-273), Washington DC: American Psychological Association.

BAUM, J.R., FRESE, M. \& BARON, R.A. (2007): The psychology of entrepreneurship, Mahwah, NJ: Erlbaum.

BEGIN, L. \& FAYOLLE, A. (2014): "Family entrepreneurship: What we know, what we need to know", en A. FAYOLLE (Ed.), Handbook of research on entrepreneurship: What we know and what we need to know (pp. 183-212), Cheltenham, UK: Edward Elgar. 
BEL DURÁN, P., FERNÁNDEZ, J., GARCÍA, C., LEJARRIAGA, G. \& MARTÍN, S. (2016): Las sociedades cooperativas de enseñanza como impulsoras de iniciativas de creación de empresas, Madrid: UCETAM.

BORNSTEIN, D. (2004): How to change the world: Social entrepreneurs and the power of new ideas, New York: Oxford University Press.

BRANDSTATTER, H. (2011): "Personality aspects of entrepreneurship: A look at five metaanalyses",Personality \& Individual Differences, 51(3),222-230, D0I:10.1016/j.paid.2010.07.007

BRETONES, F. \& RADRIGÁN, M. (2018): "Actitudes hacia el emprendimiento: El caso de los estudiantes universitarios chilenos y españoles", CIRIEC-España, Revista de Economía Pública, Social y Cooperativa, 94, 11-30, DOI: 10.7203/CIRIEC-E.94.12668

BRÜNE, N. \& LUTZ, E. (2020): "The effect of entrepreneurship education in schools on entrepreneurial outcomes: a systematic review", Management Review Quarterly, 70, 275-305, D0I: 10.1007/s11301-019-00168-3

BUSENITZ, L.W., WEST, G.P., SHEPERD, D., NELSON, T., CHANDLER, G.N. \& ZACHARAKIS, A. (2003): "Entrepreneurship research in emergence: Past trends and future directions", Journal of Management, 29, 285-308, DOI: 10.1016/S0149-2063_03_00013-8

CARLAND, J.W., HOY, F. \& CARLAND, J.A.C. (1988): "Who is an entrepreneur? Is a question worth asking?",AmericanJournal of Small Business, 12(4),33-39,D0I: 10.1177/104225878801200402

CASTRO, R.B., SANTERO, R., MARTÍNEZ, M.I. \& DE DIEGO, P. (2020): "From the economic to the social contribution of the Social Economy. Monetary assessment of the social value created for the Spanish economy", CIRIEC-España, Revista de Economía Pública, Social y Cooperativa, 100, 31-65, DOI: 10.7203/CIRIEC-E.100.18163

COSTAS, A. (2020): "Un nuevo contrato social postpandémico. El papel de la Economía Social”, CIRIEC-España, Revista de Economía Pública, Social y Cooperativa, 100, 11-29. DOI: 10.7203/ CIRIEC-E.100.18715

DACIN, M.T., DACIN, P.A. \& TRACEY, P. (2011): "Social entrepreneurship: A critique and future directions", Organization Science, 22(5), 1203-1213, D0I: 10.2307/41303113

DARMANTO, S. \& YULIARI, G. (2018): "Mediating role of entrepreneurial self-efficacy in developing entrepreneurial behavior of entrepreneur students", Academy of Entrepreneurship Journal, 24(1), 1-14, D0I: 1528-2686-24-1-128

DÁVILA, M.C., ZLOBINA, A. \& SERRANO PASCUAL, A. (2021): "Emprendimiento social en jóvenes: Análisis de su relación con otras formas de participación social", REVESCO. Revista de Estudios Cooperativos, 138, e75562, D0I: 10.5209/reve.75562 
DEES, J.G. (1998): The meaning of social entrepreneurship, disponible en https://community-wealth.org/sites/clone.community-wealth.org/files/downloads/paper-dees.pdf

DEFOURNY, J., HULGARD, L. \& PESTOFF, V. (2014): Social enterprise and the Third Sector. Changing European landscapes in a comparative perspective, London \& New York: Routledge.

DUQUE, P., MEZA, O.E., GIRALDO, D. \& BARRETO, K. (2021): “Economía Social y Economía Solidaria: un análisis bibliométrico y revisión de literatura", REVESCO. Revista de Estudios Cooperativos, 138, e75566, D0I: 10.5209/reve.75566

DURÁN-APONTE, E. \& ARIAS-GÓMEZ, D. (2015): “Intención emprendedora en estudiantes universitarios: Integración de factores cognitivos y socio-personales", Revista Colombiana de Ciencias Sociales, 6(2), 320-340, DOI: 10.21501/22161201.1528

EWEN, R. (2010): An introduction to theories of personality, Sussex: Psychology Press.

FARRINGTON, S.M. (2012): “Does personality matter for same business success?”, South African Journal of Economic \& Management Sciences, 15(4), 382-401, D0I: 10.4102/sajems.v15i4.243

FRANK, H., LUEGUER, M. \& KORUNKA, C. (2007): "The significance of personality in business start-up intentions, start-up realization and business success", Entrepreneurship \& Regional Development, 9(3), 227-251, D0I: 10.1080/08985620701218387

GALERA, G. \& BORZAGA, C. (2009): "Social enterprise: An international overview of its conceptual evolution and legal implementation", Social Enterprise Journal, 5(3), 210-228. D0I: $10.1108 / 17508610911004313$

GARCÍA-FLORES, V. \& PALMA, L. (2019): "Innovación social: Factores claves para su desarrollo en los territorios", CIRIEC-España, Revista de Economía Pública, Social y Cooperativa, 97, 245278, DOI: 10.7203/CIRIEC-E.97.14148

GARCÍA-GONZÁLEZ, A., RAMÍREZ-MONTOYA, M.S., DE LEÓN, G \& ARAGÓN, S. (2020): “El emprendimiento social como una competencia transversal: construcción y validación de un instrumento de valoración en el contexto universitario", REVESCO. Revista de Estudios Cooperativos, 136, e71862, DOI: $10.5209 /$ reve.71862

GLOBAL ENTREPRENEURSHIP MONITOR (GEM) (2019a): Reporte Nacional de Chile 2019, Santiago de Chile: Universidad del Desarrollo.

GLOBAL ENTREPRENEURSHIP MONITOR (GEM) (2019b): Comunidad Autónoma del País Vasco. Informe Ejecutivo 2019-2020, Bilbao, España: Euskal Ekintzailetzaren Behatokia-Observatorio Vasco del Emprendimiento, EEB-OVE.

GLOBAL ENTREPRENEURSHIP MONITOR (GEM) (2021): 2020/2021 Global Report, London: Global Entrepreneurship Research Association, London Business School. 
GREENE, F.J. (2021): "Stimulating Youth Entrepreneurship", en T.M. COONEY (Ed.), The Palgrave Handbook of Minority Entrepreneurship (pp. 159-178), Palgrave Macmillan.

GUPTA, P., CHAUHAN, S., PAUL, J. \& JAISWAL, M.P. (2020): “Social entrepreneurship research: A review and future research agenda", Journal of Business Research, 113, 209-229, DOI: 10.1016/j. jbusres.2020.03.032

HISRICH, R.D., LANGAN-FOX, J. \& GRANT, S. (2007): "Entrepreneurship research and practice: A call to action for psychology", American Psychologist, 62(6), 575-589, D0I: 10.1037/0003-066X.62.6.575

KESSLER, A. \& FRANK, H. (2009): "Nascent entrepreneurship in a longitudinal perspective: The impact of person, environment, resources and the founding process on the decision to start business activities", International Small Business Journal, 27(6), 720-742, DOI: $10.1177 / 0266242609344363$

KIM, A., MOON, C.W., KIM, S.K., KOH, Y.S. \& SHIN, J. (2020): "An empirical investigation on the psychological antecedents of social entrepreneurship", Entrepreneurship Research Journal, 10(3), 20170129, DOI: 10.1515/erj-2017-0129

KRUEGER, N.F., REILLY, M.D. \& CARSRUD, A.L. (2000): "Competing models of entrepreneurial intentions", Journal of Business Venturing, 15(5), 411-432, DOI: 10.1016/S08839026(98)00033-0

KRUEGER, N.F. (2003): "The cognitive psychology of entrepreneurship", en Z. ACS \& D. AUDRETSCH (Eds.), Handbook of entrepreneurial research (pp. 105-140), Oxford: Kluwer Academic Publishers.

KRUSE, P. (2020): "Can there only be one? An empirical comparison of four models on social entrepreneurial intention formation", International Entrepreneurship \& Management Journal, 16, 641-665, D0I: 10.1007/s11365-019-00608-2

MCCLELLAND, D. (1989): Estudio de la motivación humana, Madrid: Narcea.

MAIR, J. \& MARTI, I. (2006): "Social entrepreneurship research: A source of explanation, prediction, and delight", Journal of World Business, 41(1), 36-44, D0I: 10.1016/j.jwb.2005.09.002

MAIR, J. \& NOBOA, E. (2006): "Social entrepreneurship: How intentions to create a social enterprise get formed", en J. MAIR, J. ROBINSON \& K. HOCKERTS (Eds.), Social entrepreneurship (pp. 203-214), Basingstoke: Palgrave Macmillan.

MARTÍNEZ DE LUCO, G.A. \& CAMPOS, J.A. (2014): "La intención emprendedora en estudiantes universitarios: El caso de la Universidad de Deusto", Boletín de Estudios Económicos, LXIX(211), 151-172. 
MONZÓN, J.L. \& CHAVES, R. (2017): Evolución reciente de la Economía Social en la Unión Europea, Bruselas: Comité Económico y Social Europeo de la Unión Europea.

MOORE, S. \& GULLONE, E. (1996): "Predicting adolescent risk behavior using a personalized cost-benefit analysis", Journal of Youth \& Adolescence, 25(3), 343-359, D0I: 10.1007/ BF01537389

MURPHY, P.J. \& COOMBES, S.M. (2009): "A model of social entrepreneurial discover", Journal of Business Ethics, 85, 325-336, D0I: 10.1007/s10551-008-9921-y

NEWMAN, A., OBSCHONKA, M., SCHWARZ, S., COHEN, M. \& NIELSEN, I. (2018): “Entrepreneurial self-efficacy: A systematic review of the literature on its antecedents and outcomes, and an agenda for future research", Journal of Vocational Behavior, 110-B, 403-419, D0I: 10.1016/j. jvb.2018.05.012

OBSCHONKA, M., HAKKARAINEN, K., LONKA, K. \& SALMELA-ARO, K. (2017): “Entrepreneurship as a twenty-first century skill: Entrepreneurial alertness and intention in the transition to adulthood", Small Business Economics, 48, 487-501, DOI: 10.1007/s11187-016-9798-6

OLIVER, A. \& GALIANA, L. (2015): "Development and validation of the Escala de Actitudes Emprendedoras para Estudiantes (EAEE)", The Spanish Journal of Psychology, 18, E14, DOI: $10.1017 /$ sjp.2015.14

PALAMIDA, E. (2016): Determinants of entrepreneurial intentions: The interrelated role of background, situational and psychological factors, Tesis doctoral, Newcastle University.

PEATTIE, K. \& MORLEY, A. (2008): Social enterprises: Diversity and dynamics, contexts and contributions, London: Social Enterprise Coalition.

PEDROSA, I. (2015): Evaluación de la personalidad emprendedora mediante un Test Adaptativo Informatizado, Tesis doctoral, Universidad de 0viedo.

PEREDO, A.M. \& MCLEAN, M. (2006): "Social entrepreneurship: A critical review of the concept”, Journal of World Business, 41(1), 56-65, D0I: 10.1016/j.jwb.2005.10.007

PIHIE, Z.A.L. \& BAGHERI, A. (2018): "Students' entrepreneurial regulation and intention to become an entrepreneur: A comparison between public and private universities", South African Journal of Business Management, 44(4), 25-32, D0I: 10.4102/sajbm.V44i4.166

RAUCH, A. \& FRESE, M. (2007): “Let's put the person back into entrepreneurship research: A meta-analysis on the relationship between business owners' personality traits, business creation, and success", European Journal of Work \& Organizational Psychology, 16(4), 353-385, D0I: $10.1080 / 13594320701595438$ 
REYNOLDS, P.D., BYGRAVE, W.D. \& AUTIO, E. (2004): Global Entrepreneurship Monitor 2003 executive report, Babson Park, MA: Babson College.

ROTTER, J.B. (1966): “Generalized expectancies for internal versus external control of reinforcement", Psychological Monographs: General and Applied, 80(1), 1-28, DOI: 10.1037/h0092976

SÁNCHEZ, J., MARTÍN, S., BEL DURÁN, P. \& LEJARRIAGA, G. (2018): “Educación y formación en emprendimiento social: características y creación de valor social sostenible en proyectos de emprendimiento social", REVESCO. Revista de Estudios Cooperativos, 129, 16-38, D0I: 10.5209/ REVE.62492

SCHRODER, E. SCHMITT-RODERMUND, E \& ARNAUD, N. (2011): "Career choice intention of adolescents with a family business background", Family Business Review, 24(4), 305-321, D0I: 10.1177/0894486511416977

SHANE, S. \& VENKATARAMAN, S. (2000): "The promise of entrepreneurship as a field of research", Academy of Management Review, 25, 217-226, D0I: 10.2307/259271

SHANE, S. (2003): A general theory of entrepreneurship. The individual-opportunity nexus, Cheltenham: Edward Elgar.

SULLIVAN-MORT, G., WEERAWARDENA, J. \& CARNEGIE, K. (2003): "Social entrepreneurship: Towards conceptualization and measurement", International Journal of Nonprofit and Voluntary Sector Marketing, 8(1), 76-88, D0I: 10.1002/nvsm.202

SWARUPA, S.G. \& GUYAL, R.K. (2020): "Entrepreneurial intentions of students: Review of academic literature", International Journal of Scientific \& Engineering Research, 11(1), 1146-1168, DOI: $10.14299 /$ ijser.2020.01.02

TAN, W.L., WILLIAMS, J. \& TAN, T.M. (2005): “Defining the 'social' in 'social entrepreneurship': Altruism and entrepreneurship", International Entrepreneurship \& Management Journal, 1(3), 353-365, DOI: $10.1007 / \mathrm{s} 11365-005-2600-\mathrm{x}$

THOMPSON, E.R. (2009): "Individual entrepreneurial intent: Construct clarification and development of an internationally reliable metric", Entrepreneurship Theory \& Practice, 33(3), 669-694, D0I: $10.1111 / \mathrm{j} .1540-6520.2009 .00321 . \mathrm{x}$

TIWARI, P., BHAT, A.K. \& TIKORIA, J. (2017): "An empirical analysis of the factors affecting Social Entrepreneurial Intentions", Journal of Global Entrepreneurship Research, 7(1), DOI: 10.1186/s40497-017-0067-1

TRAN, A.T.P. (2018): Social entrepreneurial intention: An empirical study in Vietnam, Tesis doctoral, University of Koblenz. 
TRAN, A.T.P. \& VON KORFLESCH, H. (2018): "Social entrepreneurial intention: The case of National Economics University's students", Advance Research Journal of Multidisciplinary Discoveries, 3(1), 27-34.

VALENCIA, J.A. \& MARULANDA, F.A. (2019): “Evolución y tendencias investigativas en autoeficacia emprendedora: un análisis bibliométrico", Estudios Gerenciales, 35(151), 219-232, DOI: 10.18046/j.estger.2019.151.3277

VERHEUL, I., THURIK, R., HESSELS, J. \& ZWAN P. (2016): “Factors influencing the entrepreneurial engagement of opportunity and necessity entrepreneurs", Eurasian Business Review, 6(3), 273-295, DOI: $10.1007 / \mathrm{s} 40821-016-0065-1$

VOLKMANN, C., TOKARSKI, K.O. \& ERNST, K. (2012): "Background, characteristics and context of social entrepreneurship", en C. VOLKMANN, K.O. TOKARSKI \& K. ERNST (Eds.), Social entrepreneurs and social business. An introduction and discussion with case studies (pp. 3-30), Wiesbaden: Springer Gabler.

WANG, J.H., CHANG, C.C., YAO, S.N. \& LIANG, C. (2016): "The contribution of self-efficacy to the relationship between personality traits and entrepreneurial intention", Higher Education, 72(2), 209-224, DOI: 10.1007/s10734-015-9946-y

YAMINI, R., SOLOVEVA, D. \& PENG, X. (2020): "What inspires social entrepreneurship? The role of prosocial motivation, intrinsic motivation, and gender in forming social entrepreneurial intention", Entrepreneurship Research Journal, 20190129, 1-35, D0I: 10.1515/erj-2019-0129

YOUNG BUSINESS TALENTS (2018): Informe Young Business Talents, Business \& Marketing School-Praxis MMT.

ZAHRA, S.A., GEDOJLOVIC, E., NEUBAUM, D.O. \& SCHULAN, J.M. (2009): "A typology of social entrepreneurs: Motives, search processes and ethical challenges", Journal of Business Venturing, 24(5), 519-532, DOI: 10.1016/j.jbusvent.2008.04.007

ZHAO, H., SEIBERT, S.E. \& LUMPKIN, G.T. (2010): "The relationship of personality to entrepreneurial intentions and performance: A meta-analytic review", Journal of Management, 36(2), 381-404, DOI: 10.1177/0149206309335187 